\title{
Criminologie
}

\section{Équivalences pénales et solutions de rechange à l'emprisonnement : la métrique pénale implicite des tribunaux criminels}

\author{
Pierre Tremblay, Sylvie Gravel et Maurice Cusson
}

Volume 20, numéro 2, 1987

La détermination de la sentence

URI : https://id.erudit.org/iderudit/017252ar

DOI : https://doi.org/10.7202/017252ar

Aller au sommaire du numéro

Éditeur(s)

Les Presses de l'Université de Montréal

ISSN

0316-0041 (imprimé)

1492-1367 (numérique)

Découvrir la revue

Citer cet article

Tremblay, P., Gravel, S. \& Cusson, M. (1987). Équivalences pénales et solutions de rechange à l'emprisonnement : la métrique pénale implicite des tribunaux criminels. Criminologie, 20(2), 69-88. https://doi.org/10.7202/017252ar
Résumé de l'article

Sentencing research has generally neglected taking into account how perceptions of the severity of available sanctions affect sentencing behavior. Based on the magnitude estimates of just how severe penalties of different kinds and different amounts criminal courts perceive them to be, this paper identifies the current exchange rates on what Wilkins has called the "punishment market" explains why so many alternatives to imprisonment experiments have backfired, offers a justification for the existing penal repertoire and finds criminal court's severity scales to be socially well grounded. 


\section{ÉQUIVALENCES PÉNALES ET SOLUTIONS DE RECHANGE}

À L'EMPRISONNEMENT:

LA MÉTRIQUE PÉNALE IMPLICITE DES TRIBUNAUX CRIMINELS

Pierre Tremblay*, Sylvie Gravel**, Maurice Cusson***

Sentencing research has generally neglected taking into account how perceptions of the severity of available sanctions affect sentencing behavior. Based on the magnitude estimates of just how severe penalties of different kinds and different amounts criminal courts perceive them to be, this paper identifies the current exchange rates on what Wilkins has called the "punishment market", explains why so many alternatives to imprisonment experiments have backfired, offers a justification for the existing penal repertoire and finds criminal court's severity scales to be socially well grounded.

\section{INTRODUCTION}

Toute sentence criminelie présuppose implicitement qu'une certaine correspondance existe entre l'intensité objective de punition infligée (exprimable en jours de prison ou dollars d'amende par exemple) et le degré de sévérité subjective que l'on cherche à atteindre en dosant la sentence de la sorte. L'ensemble de ces correspondances constitue l'objet d'étude de ce qu'on pourrait appeler la «métrique pénale». Un certain nombre de techniques ont été mises au point pour mesurer la sévérité perçue des peines que les tribunaux infligent aux délinquants pour les crimes qu'ils ont commis (Sebba, 1978; Erickson et Gibbs, 1979; Buchner, 1979; Sebba et Nathan, 1984; McClelland et Alpert, 1985; Allen et Benson, 1985).

Nous commencerons par analyser la métrique pénale qu'utilisent implicitement (inconsciemment) les acteurs judiciaires (juges, avocats de

1. Cet article résulte d'une recherche en cours sur le jugement pénal, subventionnée par le Conseil de recherches en sciences humaines du Canada, le Fonds pour la formation de chercheurs et l'aide à la recherche, le solliciteur général et le ministère de la Justice du Canada.

Cet article est une version remaniée d'un rapport remis en avril 1987 à la division de recherche du ministère de la Justice intitulé "Métrique pénale, alternatives à l'emprisonnement et l'avenir des agents de probation*.

* Professeur au Département de sociologie de l'université McGill à Montréal.

** Attachée de recherche au Centre international de criminologie comparée de l'Université de Montréal.

*** Professeur à l'École de criminologie de l'Université de Montréal. 
la défense, procureurs de la Couronne et agents de probation). On s'intéressera aux propriétés structurelles des peines elles-mêmes (emprisonnement, amende, probation et travaux communautaires) et au consensus social qui caractérise la manière dont on perçoit leur sévérité relative. La deuxième section de cet article, plus appliquée, examine le «taux de change» actuel sur le marché des peines, spécifie le champ d'application légitime des peines substitutives à l'emprisonnement, et démontre comment ce taux de change structure les choix sentenciels auxquels sont confrontés quotidiennement les tribunaux criminels.

\section{DONNÉES}

Les échelles de sévérité pénale que nous examinons ici proviennent d'une enquête auprès des 403 juristes et professionnels qui en 1986 participaient directement ou indirectement au processus sentenciel des tribunaux criminels du palais de justice de Montréal2: 90 juges, 191 avocats de la défense, 75 procureurs de la Couronne et 47 agents de probation. La participation au sondage a été de $62 \%$ chez les avocats, $64 \%$ chez les procureurs et $76 \%$ pour les agents de probation. La participation des magistrats à l'enquête a été plutôt faible ( $36 \%$ seulement), les juges de la Cour supérieure (Division criminelle) ayant refusé, par principe, d'être interviewés ( Un juge ne devant s'exprimer que par ses jugements"). Par contre, 53\% des juges des Sessions de la paix et 79\% des magistrats de la Cour municipale ont accepté de compléter l'entrevue. Ces deux instances judiciaires acheminent, comme on le sait, la très grande majorité des causes criminelles ${ }^{3}$.

On évalue généralement la sévérité perçue des peines en demandant aux répondants d'indiquer leur degré de sévérité sur une échelle de 0 à 100. Mais rien ne nous garantit qu'une peine recevant un score de 20 soit effectivement perçue comme étant deux fois plus sévère qu'une peine recevant un score de 10. Les intervalles d'une telle échelle ne sont pas généralement égaux. Elle a également l'inconvénient de tronquer la distribution des jugements en fixant artificiellement un score maximal. C'est pourquoi nous avons préféré demander aux répondants eux-mêmes de déterminer le degré de sévérité d'une série de peines en les comparant chaque fois à la sévérité d'un standard ou d'un module servant de point de

2. L'enquête a été réalisée en $\mathbf{1 9 8 6}$ par une dizaine d'interviewers de la maison de sondage CROP. La durée de l'entrevue a été d'une heure et demie en moyenne. Les répondants ont été interviewés à leurs bureaux sur rendez-vous, après avoir reçu préalablement une lettre circulaire leur indiquant la nature de l'enquête.

3. Dans cet article nous nous intéressons au comportement de l'ensemble des acteurs judiciaires interviewés, chaque catégorie d'acteur recevant le même poids statistique. 
comparaison. Le module utilisé a été «un an de prison». Les sujets interviewés avaient à indiquer si les stimuli pénaux qu'on leur soumettait successivement étaient moins sévères, aussi sévères ou plus sévères qu'un an de prison et jusqu'à quel point ${ }^{4}$. On obtient ainsi ce que les psychophysiciens appellent une échelle d'amplitude.

La théorie méthodologique de ces échelles d'amplitude (Stevens, 1975; Hamblin, 1974; Shinn, 1974; Lodge, 1981) repose sur deux hypothèses bien établies: a) l'intensité ressentie de la sensation que génère un stimulus quelconque, physique ou social, n'est pas une fonction linéaire mais une fonction puissance de l'intensité du stimulus lui-même (une peine de quatre ans de prison est objectivement deux fois plus lourde qu'une peine de deux ans de prison, mais elle sera perçue, nous le verrons, comme beaucoup plus sévère) ${ }^{s} ; b$ ) les rapports d'intensité perçus entre diverses grandeurs d'un même phénomène peuvent être évalués directement par les sujets eux-mêmes en leur faisant exécuter une série de comparaisons par rapport à un stimulus standard. Les scores d'amplitude générés par ces comparaisons peuvent être alors échelonnés sur une "vraie" échelle d'intensité, c'est-à-dire une échelle à intervalles égaux et sur laquelle les opérations de division ou de multiplication sont admissibles. Si le score de sévérité d'une amende de $100 \$$ est de 10 , et que le score d'une probation de 10 mois est de 30 , on peut être alors assuré que cette sentence de probation est effectivement perçue comme étant "trois fois plus sévère" (Cf. tableau 1).

Mentionnons, pour finir, que les stimuli pénaux, contrairement à d'autres stimuli (par exemple la gravité des crimes), sont "métriques» au départ: un an de prison, 120 heures de travaux communautaires, etc. Deux sortes de biais sont possibles. Les sujets peuvent être enclins à donner une réponse «arithmétique» plutôt qu'une estimation subjective d'intensité; trois ans de prison seraient "trois fois" plus sévères qu'un an de prison, tout simplement parce qu'un an de prison est objectivement une peine trois fois moins longue à endurer qu'une peine de trois ans. Deuxième sorte de biais: la réponse "mémorisée», où le sujet estime la sévérité de la peine $\mathrm{A}$ en tenant compte de son estimation de la peine $\mathrm{B}$,

4. Les répondants avaient simplement à comparer la sévérité des peines qu'il leur était présentée à celle d'une année de prison. Aucune valeur n'était assignée à ce module de référence et ce n'est qu'à l'étape de la codification des données que le module a reçu la valeur de 100 points. Les scores de sévérité perçus ont été alors calculés sur cette base.

5. C'est pour cette raison que la mesure de tendance centrale pour ce genre de données est la moyenne géométrique et pas la moyenne arithmétique (Lodge, 1981). Celle-là se base en effet sur la puissance moyenne à laquelle les répondants ont élevé le module ou l'étalon de référence. 
évaluée précédemment. Pour minimiser les réponses mémorielles et les réponses arithmétiques, les stimuli sont notés sur des cartes, un stimulus par carte, et l'interviewer reçoit la consigne de retourner chaque carte immédiatement après la réponse et de ne pas autoriser le sujet à "revenir en arrière" pour "vérifier» le score d'un stimulus antérieur ou le "corriger». Les peines de même nature ont été présentées de manière que les stimuli de petite intensité succèdent aux stimuli d'intensité élevée et qu'entre deux peines de même nature s'intercalent un nombre maximum de peines qualitativement différentes.

\section{LES PROPRIÉTÉS STRUCTURELLES DES PEINES}

Les tribunaux criminels ont toujours utilisé simultanément un répertoire varié de sanctions très différentes les unes des autres pour réprimer les crimes. L'analyse de la sévérité perçue des peines les plus couramment utilisées par les tribunaux criminels contemporains (l'emprisonnement, l'amende, la probation et les travaux communautaires) constitue une voie d'accès privilégiée à l'intelligibilité latente du comportement quotidien de la justice criminelle dans son ensemble.

La figure 1 présente les échelles de sévérité perçues d'un certain nombre de sanctions criminelles telles qu'elles sont perçues par les tribunaux criminels étudiés. L'axe vertical représente les scores moyens de sévérité assignés aux divers stimuli pénaux présentés aux répondants (100 étant la valeur que nous avons convenu d'attribuer à un an d'emprisonnement). L'axe horizontal indique la quantité objective de punition infligée en jours (de prison ou de probation) et en dollars (pour les amendes). Puisqu'une journée de travail est de 8 heures, l'équivalent d'une journée pénale de travaux communautaires est de 8 heures plutôt que de $\mathbf{2 4}$ heures comme c'est le cas pour les journées de prison ou de probation.

Les diverses peines auxquelles ont recours les tribunaux criminels peuvent être évaluées en fonction de leur sévérité marginale ou de leur sévérité intrinsèque. La sévérité «intrinsèque» d'une peine dépend de la nature du bien auquel elle porte atteinte. Il est normal (dans les sociétés modernes occidentales) qu'une peine privative de liberté (l'emprisonnement) soit perçue - à intensité égale - comme intrinsèquement plus sévère qu'une peine patrimoniale (l'amende) ou restrictive de liberté. La sévérité "marginale" d'une peine est la sévérité additionnelle qu'une quantité supplémentaire de punition procure. Un mois de prison est perçu comme ayant le même degré de sévérité qu'une amende de $1223 \$$. Alors que trois mois de prison est considéré comme une peine de 2,47 fois plus 


\section{FIGURE I}

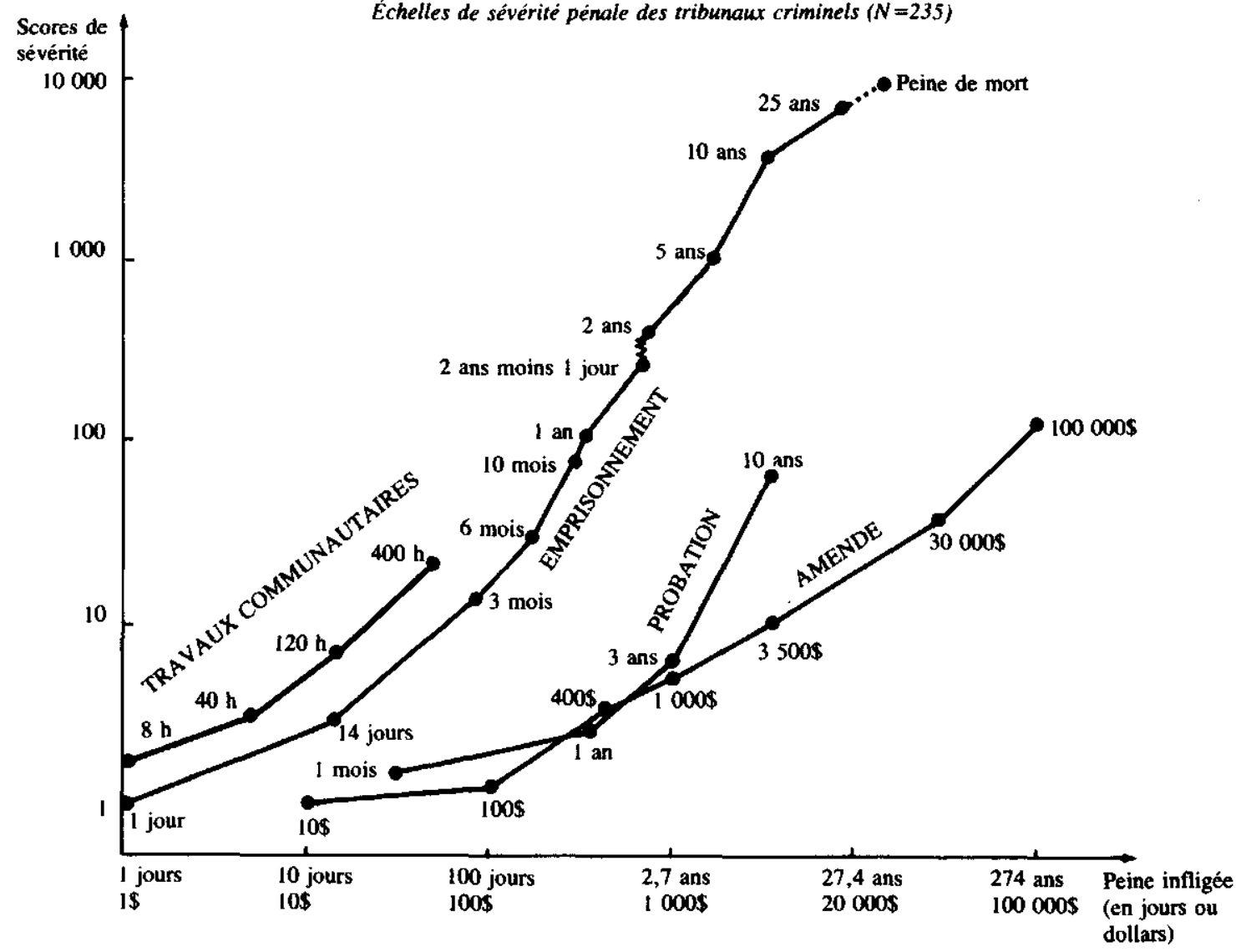


sévère qu'un mois, une amende objectivement trois fois plus élevée (3 000\$) est perçue comme étant simplement 1,7 fois plus sévère qu'une amende de $1000 \$$. La sévérité marginale des peines d'emprisonnement est par conséquent supérieure à celle des peines patrimoniales.

Les peines qu'imposent les tribunaux criminels contemporains peuvent être ainsi évaluées non seulement en fonction de leur sévérité marginale (faible ou élevée, constante ou variable) mais également en fonction de leur sévérité intrinsèque (faible ou élevée). Ces propriétés structurelles des peines peuvent être spécifiées empiriquement en procédant à une analyse statistique de chacune des quatre échelles de sévérité que la figure 1 présente 6 .

Le tableau 2 présente les résultats de cette analyse ${ }^{7}$ et suggère qu'il est possible de classer adéquatement les diverses peines couramment utilisées par les tribunaux criminels contemporains selon leur niveau de sévérité intrinsèque et leur degré de sévérité marginale. Un résultat instructif à divers points de vue. Newman (1983) propose une description élaborée de la nature des souffrances imposées (souffrances chroniques ou aiguës), de leurs impacts à court ou moyen terme (impacts économiques, sociaux, mentaux) ou des risques de victimisation qu'elles peuvent produire. Une approche positiviste qui ne parvient pas à prođuire une typologie acceptable des peines pour diverses raisons. Les coûts de la peine varient selon les condamnés; des peines différentes ont des conséquences souvent identiques; et à privilégier une définition stricte de la "souffrance" (comme souffrance physique) la plupart des sanctions couramment utilisées par les tribunaux apparaissent comme des pseudochâtiments (la probation) ou des peines inclassables (l'amende). La perspective que nous proposons ici se base au contraire sur la manière dont ceux qui punissent (les tribunaux criminels) ou qui demandent qu'une punition soit imposée (la société civile) perçoivent la sévérité des

6. Étant donné que la perception d'un stimulus n'est pas une fonction linéaire mais une fonction puissance de son intensité (Stevens, 1975) le modèle d'analyse de la relation entre la sévérité perçue des peines et leur intensité objective est $: \log \mathrm{Y}=\mathbf{a}+\mathbf{b} \log \mathrm{X}+\mathrm{e}$ où $\mathrm{Y}=$ la moyenne géométrique des scores de sévérité à un stimulus pénal par les répondants et $\mathrm{X}=$ la quantité de jours (de prison, de probation, de travail communautaire) ou de dollars de punition infligée. Ce modèle est également celui qu'ont utilisé Erickson et Gibbs (1979), Sebba et Nathan (1985) ou McClelland et Alpert (1986).

7. Nous avons procédé à une analyse de régression des 4 échelles de sévérité pénale de la figure: prison $(N=8)$, amende $(N=5)$, probation $(N=4)$ et travaux communautaires $(\mathrm{N}=4)$. La sévérité marginale d'une peine est mesurée par la pente ou le coefficient «b" de l'équation de régression. La sévérité intrinsèque d'une peine est mesurée par la constante "a a de cette même équation. Les paramètres «a» et «b* de chaque échelle pénale analysée dans cette étude sont: amende $(a=0,801, b=0,536)$, probation $(a=-1,112, b=0,719)$, travaux communautaires $(a=0,145, b=0,629)$ et prison $(a=-0,496, b=1,026)$. 
peines infligées. Les résultats du tableau 2 suggèrent que chaque sorte de peine a son utilité propre (une combinaison spécifique de sévérité marginale et de sévérité intrinsèque) et que les tribunaux criminels exploitent effectivement un répertoire pénal en quelque sorte "complet». La peine capitale constitue la forme limite d'une peine ayant une sévérité intrinsèque absolue. Les châtiments corporels, d'autre part, ont pour caractéristique fondamentale de posséder une sévérité marginale absolue. L'approche "perceptuelle» révèle ainsi l'intelligibilité latente des propriétés structurelles des peines. Elle nous fait comprendre notamment pourquoi les tribunaux criminels ont toujours privilégié un régime pénal multiple plutôt que purement carcéral ou purement patrimonial par exemple. Elle nous fait également comprendre que les peines de probation, d'amende ou de travaux communautaires ne doivent pas être conçues uniquement en termes négatifs (comme des peines "non carcérales" ou comme n'étant pas "vraiment» des peines).

Cette typologie n'a pas seulement une validité idiosyncrasique (spécifique au palais de justice enquêté). Une analyse des propriétés structurelles des peines basée sur les échelles de sévérité pénale d'un échantillon représentatif de la société civile montréalaise aboutit aux mêmes résultats (Tremblay, 1987). Les résultats obtenus par Sebba et Nathan (1984) ou McClelland et Alpert (1985), à partir d'échantillons très différents (étudiants, policiers, détenus), témoignent également de la robustesse de cette typologie.

\section{CONSENSUS SOCIAL ET MÉTRIQUE PÉNALE}

Dans cette section, nous examinons s'il existe en matière de sévérité perçue des peines un consensus entre la société civile et les tribunaux criminels. Les problèmes méthodologiques particuliers que pose l'analyse du consensus social ont également fait l'objet de mises au point dont nous nous inspirons ici (Jacobsen et Van der Voordt, 1980; Miethe, 1984; Rossi et Berk, 1985). Les échelles de sévérité des peines de la société civile à laquelle s'adressent les tribunaux criminels étudiés dans cet article proviennent d'un sondage représentatif de la population francophone adulte de la région métropolitaine de Montréal $(N=299)^{8}$. Pour évaluer s'il existe un consensus quant à la manière dont les citoyens

8. Le questionnaire administré était le même que celui auquel ont répondu les acteurs judiciaires et les échelles de sévérité perçues des peines, obtenues exactement de la même façon. Le sondage auprès du public a été réalisé 6 mois avant l'enquête auprès des tribunaux criminels. Pour une description et une analyse pius détaillée de la métrique pénale utilisée implicitement par le public, voir Tremblay, 1987. 
TABLEAU 1

Scores de sévérité perçus des peines des tribunaux criminels $(N=235)$

\begin{tabular}{|c|c|c|c|c|c|c|}
\hline $\begin{array}{l}\text { Sévérité relative du } \\
\text { stimulus par rapport } \\
\text { à un an de prison }\end{array}$ & Stimulus pénal & $\begin{array}{l}\text { Ordre de } \\
\text { présentation } \\
\text { du stimulus }\end{array}$ & $\begin{array}{l}\text { Moyenne } \\
\text { géométrique }\end{array}$ & Médiane & $\begin{array}{l}\text { Moyenne } \\
\text { arithmétique }\end{array}$ & $\begin{array}{l}\text { Coefficient } \\
\text { de variation } \\
(\%)\end{array}$ \\
\hline 147 fois plus sévère & Peine de mort & 1 & 14689 & 10015 & 36226 & 175 \\
\hline 64 fois plus sévère & $\begin{array}{l}\text { Pénitencier } \\
25 \text { ans }\end{array}$ & 3 & 6412 & 8002 & 8469 & 101 \\
\hline 23 fois plus sévère & $\begin{array}{l}\text { Pénitencier } \\
10 \text { ans }\end{array}$ & 19 & 2270 & 2472 & 3216 & 83 \\
\hline 9,5 fois plus sévère & $\begin{array}{l}\text { Pénitencier } \\
5 \text { ans }\end{array}$ & 14 & 953 & 734 & 1601 & 128 \\
\hline 3,5 fois plus sévère & $\begin{array}{l}\text { Pénitencier } \\
2 \text { ans } \\
\text { Prison } 2 \text { ans }\end{array}$ & 5 & 348 & 256 & 701 & 179 \\
\hline 2,5 fois plus sévère & moins 1 jour & 21 & 258 & 208 & 431 & 156 \\
\hline 1,2 fois plus sévère & $\begin{array}{l}\text { Amende } 100000 \$ \\
\text { Prison } 1 \text { an }\end{array}$ & $\begin{array}{c}8 \\
\text { Modulus }\end{array}$ & $\begin{array}{l}123 \\
100\end{array}$ & 100 & 548 & 190 \\
\hline 1,4 fois moins sévère & Prison 10 mois & 11 & 73,11 & 97,1 & 78,5 & 34 \\
\hline 1,5 fois moins sévère & Probation 10 ans & 22 & 65,31 & 100,0 & 375 & 196 \\
\hline 2,7 fois moins sévère & Amende $30000 \$$ & 28 & 37,41 & 50,0 & 237 & 262 \\
\hline 3,6 fois moins sévère & Prison 6 mois & 7 & 27,48 & 49,9 & 37,8 & 47 \\
\hline 5 fois moins sévère & Trav. comm. $400 \mathrm{~h}$ & 18 & 19,82 & 24,9 & 65,1 & 143 \\
\hline
\end{tabular}


7,2 fois moins sévère 9,9 fois moins sévère 14,9 fois moins sévère 15,7 fois moins sévère 19,4 fois moins sévère 25 fois moins sévère 31,4 fois moins sévère 32,8 fois moins sévère 33,4 fois moins sévère 37 fois moins sévère

60 fois moins sévère 68 fois moins sévère 79 fois moins sévère 100 fois moins sévère 101 fois moins sévère
Prison 3 mois

Amende 3500 s

Trav. comm. $120 \mathrm{~h}$

Probation 3 ans

\section{Amende 1000 s}

Probation 6 mois

Amende 400\$

Trav. comm. $40 \mathrm{~h}$

Prison 14 jours

Probation I an

Trav. comm. 8 h

Probation 1 mois

Amende $100 \$$

Prison I jour

Amende 10 s

\section{6}

20

13

4

12

9

6

24

23

26

10

15

25

27

2
13,90

10,09

6,73

6,34

5,14

3,98

3,18

3,05

2,99

2,67

1,67

1,47

1,27

1,01

0,99
24,9

10,0

19,3

32,3

16,3

31,5

12,8

11,1

8,5

8,2

4,6

8,4

2,9

2,1

2,0

1,3

5,1 
"ordinaires» et les acteurs judiciaires apprécient ou évaluent la sévérité des peines infligées par la justice criminelle, nous avons comparé leurs échelles pénales et déterminé si elles différaient significativement les unes par rapport aux autres9.

Les résultats du tableau 3 indiquent que le public et les tribunaux criminels s'entendent non seulement sur la manière de classer les peines (par ordre croissant de sévérité) mais sur la sévérité elle-même d'une multiplicité de peines de nature différente et d'intensité variable. Ce n'est pas, non plus, uniquement sur la sévérité des peines carcérales sur lesquelles on s'accorde mais aussi sur la sévérité des peines patrimoniales et des peines de probation. Seuls les travaux communautaires font l'objet d'une divergence d'évaluation significative entre le public et les tribunaux criminels. Les tribunaux considèrent cette sorte de sanction comme étant beaucoup plus sévère que ne l'estime la société civile. Il en résulte que toute stratégie d'alternative d'emprisonnement centrée sur cette forme particulière de mesure pénale risque d'être discréditée, la demande pénale de la société civile ne pouvant être guère satisfaite de l'offre que lui proposent les tribunaux criminels.

Le consensus social absolu qui caractérise la perception de la sévérité de la plupart des peines infligées par les tribunaux criminels mérite d'être souligné 10 . Il s'agit là d'un fait pénologique fondamental qui nous renseigne tout d'abord sur la fermeté sous-jacente des structures normati-

9. Plus spécifiquement, nous avons procédé à une analyse de régression des échelles de sévérité pénale du public (variable dépendante); les scores moyens de sévérité attribués par les acteurs judiciaires constituant les valeurs de la variable indépendante. L'argument qui sous-tend l'analyse statistique présentée au tableau 3 est le suivant. S'il existait un consensus absolu ou total entre le public et les tribunaux, leurs échelles pénales seraient identiques. Dans une telle hypothèse, la constante " $a$ " et la pente " $b$ " des équations de régression auraient des valeurs de 0 (pour la constante) et de 1 (pour la pente). Pour déterminer si la valeur des paramètres a et b estimés s'écartent significativement des valeurs que postule l'hypothèse nulle d'un consensus absolu ( $a=0$ et $b=1$ ), un test de variance basé sur la statistique $F$ est utilisé, comme le suggère Miethe (1984) sur la base des travaux de Namboodiri et coll. (1975).

10. Même si la société civile et les tribunaux criminels s'entendent collectivement sur la sévérité relative des peines infligées aux délinquants, la variabilité des perceptions individuelles est importante comme en témoigne les mesures de dispersion qui caractérisent les scores de sévérité rapportés au tableau 1 (infra.) Cette variabilité - qui fera l'objet d'une analyse en soi dans une publication subséquente - est déterminée en partie par la nature des peines elles-mêmes. Il est plus facile au niveau individuel de s'entendre sur la sévérité des peines carcérales (coefficient moyen de variation de $97 \%$ ) que sur la sévérité des peines patrimoniales, des peines de travaux communautaires et de probation (coefficients moyens de variation de $203 \%, 147 \%$ et de $171 \%$ respectivement). Une deuxième source de variabilité - sur laquelle nous ne nous attarderons pas non plus dans cet article résulte du rôle stratégique qu'occupe chacun des acteurs professionnels de la scène judiciaire. 
TABLEAU 2

Propriétés structurelles des peines telles que persues par les tribunaux criminels montréalais

\begin{tabular}{llll}
\hline & & \multicolumn{2}{c}{ Sévérité marginale } \\
& Faible & Faible & Élevée \\
Sévérité absolue & Élevée & $\begin{array}{l}\text { Travail } \\
\text { communautaire }\end{array}$ & Probation \\
\hline
\end{tabular}

\section{TABLEAU 3}

Analyse de consensus (corrélation et régression) entre les échelles de sévérité pénale du public et des tribunaux criminels, par type de peine ${ }^{l}$

\begin{tabular}{|c|c|c|c|c|}
\hline Peines & $\begin{array}{l}\text { Nombre de } \\
\text { stimuli } \\
\text { pénaux } \\
\text { (N) }\end{array}$ & $r$ & $\begin{array}{l}\text { Équation de } \\
\text { régression }{ }^{2}\end{array}$ & $\begin{array}{l}\text { Test de signi- } \\
\text { fication }\end{array}$ \\
\hline $\begin{array}{l}\text { Toutes les } \\
\text { peines }\end{array}$ & $(25)$ & 0,98 & $\begin{array}{r}Y_{p}=-0,085+1,05 X_{j} \\
(0,08) \quad(0,04)\end{array}$ & $\begin{array}{c}F_{2,23}=0,68 \\
\text { (consensus) }\end{array}$ \\
\hline Emprisonnement & (10) & 0,98 & $\begin{array}{r}Y_{p}=-0,31+1,15 X_{j} \\
(0,20) \quad(0,08)\end{array}$ & $\begin{array}{c}F_{2,8}=1,98 \\
\text { (consensus) }\end{array}$ \\
\hline Amende & (6) & 0,99 & $\begin{array}{r}Y_{p}=-0,06+1,18 X_{j} \\
(0,09)\end{array}$ & $\begin{array}{c}F_{2,4}=3,83 \\
\text { (consensus) }\end{array}$ \\
\hline Probation & (4) & 0,99 & $\begin{array}{r}Y_{p}=0,11+0,98 X_{j} \\
(0,10) \quad(0,09)\end{array}$ & $\begin{array}{c}\mathrm{F}_{2,2}=1,51 \\
\text { (consensus) }\end{array}$ \\
\hline $\begin{array}{l}\text { Travaux } \\
\text { communautaires }\end{array}$ & (4) & 0,98 & $\begin{array}{r}Y_{p}=-0,05+0,54 X_{j} \\
(0,07) \quad(0,06)\end{array}$ & $\begin{array}{c}\mathrm{F}_{2,2} \hat{\mathbf{1}} 81,34^{3} \\
\text { (dissensus) }\end{array}$ \\
\hline
\end{tabular}

1 Public $(\mathrm{N}=299)$; Tribunaux criminels $(\mathrm{N}=235)$.

2 Dans chaque équation de régression $Y_{p}=$ scores de sévérité moyens du public et $X_{j}=$ scores de sévérité moyens des tribunaux criminels. Les erreurs types (erreurs standards) des paramètres a et b sont indiquées entre parenthèses. Les données traitées ne sont pas les scores bruts mais les scores transformés (moyenne des logarithmes des scores de sévérité).

${ }^{3}$ Dissensus significatif, $\mathrm{p}<0,05(\mathrm{~F}>19)$ : rejet de $\mathrm{H}_{\mathrm{o}}$. 
ves sociales, comme le démontre également le consensus social substantiel dont, par exemple, la gravité des crimes ou le prestige social des professions font l'objet (Wolfgang et coll., 1986; Rossi et Berk, 1985). Un tel consensus suggère également que la manière dont les tribunaux évaluent la sévérité des peines qu'ils infligent n'exprime pas les préférences particulières des juristes ou ne résulte pas d'une convention intrinsèquement arbitraire ou modulée par les contingences organisationnelles des palais de justice mais constitue bien au contraire un fait social, largement inconscient, de droit naturel (Cusson, 1987). On pourrait être tenté d'attribuer aux tribunaux criminels la capacité d' "influencer" directement ou indirectement (à travers les canaux médiatiques) les perceptions de sévérité des citoyens. Mais la nature inconsciente de toute métrique pénale suggère qu'une telle hypothèse est peu vraisemblable.

\section{ÉQUIVALENCES PÉNALES ET SOLUTIONS DE RECHANGE À L'EMPRISONNEMENT}

Il est possible qu'une mauvaise appréciation de la valeur des peines, de leur "prix» en termes de sévérité et des règles élémentaires de métrique pénale explique en partie les difficultés que connaissent les stratégies d'alternatives à l'emprisonnement et qui sont rapportées dans la littérature (Austin et Krisberg, 1982; Commission Archambault, 1987; Pease, 1985; Hudson et Chesney, 1978; Simon et Vallières, 1982). Connaissant, par contre, la sévérité perçue des diverses peines couramment utilisées par les tribunaux, il devient possible de les comparer entre elles, de connaître assez précisément le "taux de change" en cours sur le marché des peines et de spécifier le champ d'application «légitime» ou acceptable des mesures pénales non carcérales. Dans cette section, nous examinerons les taux de change que les tribunaux criminels établissent entre l'emprisonnement, la probation, l'amende et les travaux communautaires. Dans la section suivante, nous analyserons comment ces taux de change structurent en partie leurs choix sentenciels dans des causes criminelles particulières.

La sévérité d'une peine n'étant pas proportionnelle à son intensité objective (temps de probation ou montant pécuniaire), les équivalences ponctuelles que l'on peut établir en termes de sévérité entre peines qualitativement distinctes varient d'un niveau de sévérité à l'autre. On doit donc se baser sur les rapports qui existent entre les échelles de sévérité propres à chaque sorte de peine pour déterminer ces équivalences. Erickson et Gibbs (1979) procèdent sur la base des estimations fournies par les équations de régression qui spécifient la pente et la 
constante de chacune des échelles. De telles estimations ne sont adéquates que si les échelles sont toutes parfaitement linéaires. La figure 1 montre qu'elles ne le sont pas et que même si ces équations peuvent décrire adéquatement leurs caractéristiques structurelles générales, elles ne conviennent pas lorsqu'il s'agit d'établir des équivalences relativement précises entre quantités de punition qualitativement différentes. Nous avons donc préféré l'approche plus artisanale (mais plus fiable) de la "méthode graphique». Sachant, par exemple, qu'un mois de prison a un score de sévérité de 5,62 on obtient alors la quantité d'amende qui sur l'échelle correspond à un tel score (en l'occurrence 1 223\$). Connaissant, à l'inverse, le score de sévérité d'une amende de $200 \$$ (un score de sévérité de 2 points), on calcule alors la quantité d'emprisonnement qui sur l'échelle correspond à un tel score (en l'occurrence 5 jours).

\section{TABLEAU 4}

Équivalences pénales, telles qu'établies par les tribunaux criminels (montréalais)

\begin{tabular}{|c|c|c|c|c|}
\hline $\begin{array}{l}\text { Score de } \\
\text { sévérité perçue } \\
\text { (moyenne géon }\end{array}$ & Prison & $\begin{array}{r}\text { Amende } \\
(\$)\end{array}$ & Probation & $\begin{array}{l}\text { Travail } \\
\text { communautaire }\end{array}$ \\
\hline 0.99 & 1 jour & (49) & $(1.5 \mathrm{sem})$. & ( 2,2 heures) \\
\hline 1,05 & 1.1 jour & $(52)$ & (1,6 sem.) & (3 heures) \\
\hline 1.53 & 2,8 jours & 137 & 1 mois & $(6,1$ heures $)$ \\
\hline 1,67 & 3,7 jours & 163 & 1,7 mois & 8 heures \\
\hline 2,00 & 5 jours & 200 & 3,3 mois & 13 heures \\
\hline 2,37 & 7,5 jours & 266 & 6,7 mois & 20 heures \\
\hline 2,56 & 8,9 jours & 282 & 8,4 mois & 23 heures \\
\hline 2,99 & 14 jours & 376 & 1,1 année & 39 heures \\
\hline 3.98 & 20 jours & 631 & 1,6 année & 58 heures \\
\hline 5,62 & 1 mois & 1223 & 2,4 années & 92 heures \\
\hline 7,07 & 1,3 mois & 1884 & 3,1 années & 127 heures \\
\hline 7,50 & 1.4 mois & 2113 & 3,3 années & 134 heures \\
\hline 9,72 & 2 mois & 3548 & 3,7 années & 179 heures \\
\hline 11,86 & 2,5 mois & 4732 & 4,1 années & 225 heures \\
\hline 13,33 & 2.9 mois & 5623 & 4,3 années & 253 heures \\
\hline 13.90 & 3 mois & 6131 & 4,5 années & 268 heures \\
\hline 17,78 & 4 mois & 9173 & 5 années & 357 heures \\
\hline 23.71 & 5 mois & 14962 & 5,8 années & 477 heures \\
\hline 27,48 & 6 mois & 21135 & 6,7 années & 600 heures \\
\hline 44,67 & 8 mois & 37584 & 8,2 années & - \\
\hline 73,11 & 10 mois & 59566 & 10,3 années & - \\
\hline 89,13 & 11 mois & 70795 & 11,6 années & - \\
\hline 100 (module) & 12 mois & 84140 & 12,6 années & - \\
\hline 122,7 & 13 mois & 100000 & 13,7 années & - \\
\hline 177,8 & 18 mois & 158489 & - & - \\
\hline
\end{tabular}


Le tableau 4 présente une série d'équivalences établies sur la base des perceptions que les tribunaux criminels (montréalais) ont de leur sévérité relative. Les peines carcérales étant intrinsèquement et marginalement plus sévères que toutes les autres sortes de peines examinées dans cette étude, il était inutile, à toutes fins pratiques, d'établir ces équivalences au-delà d'un certain seuil de sévérité (en l'occurrence un score de sévérité pénale équivalent à 13 mois de prison). Les équivalences indiquées entre parenthèses dans le tableau 4 ont été obtenues non pas graphiquement mais par extrapolation. D'une part, en effet, les peines inférieures à un mois de probation et huit heures de travaux communautaires n'ont pas été inclues dans l'échantillon des stimuli pénaux soumis aux répondants. D'autre part, la capacité de différencier le degré de sévérité relative des amendes inférieures à $100 \$$ fait problème (en raison sans doute de la sévérité relativement élevée du module choisi pour construire ces échelles, soit un an de prison). Sachant ainsi que 2,8 jours de prison est perçu comme équivalent (en termes de sévérité) à une amende de $137 \$$, il en résulte (par extrapolation) qu'un jour de prison serait perçu comme aussi sévère qu'une amende de $49 \$(137 / 2,8)$. Puisque huit heures de travaux communautaires est une peine aussi sévère qu'un emprisonnement de 3,7 jours, on peut estimer qu'un jour d'emprisonnement est perçu comme une peine aussi sévère que 2,2 heures de travaux communautaires $(813,7)$, ou encore qu une heure de travaux communautaires serait une peine équivalente à une amende de $23 \$$.

Ces équivalences extrapolées semblent assez réalistes. Une analyse récente des emprisonnements par défaut de paiement d'amende dans la province de Québec indique l'équivalence "pratique " que le système de justice pénale établit entre une peine d'emprisonnement et une amende. Les prisons du Québec reçoivent approximativement 10000 admissions pour défaut de paiement d'amende par année. Ces individus ont été condamnés en moyenne à payer $100 \$$ à $110 \$$ d'amende et ils ont à purger en moyenne 2,8 jours de prison (Cousineau et coll., 1986). Un jour de prison équivaut ainsi, selon le barème informel en vigueur dans le système de justice criminelle, à une amende de $38 \$$ - une équivalence assez proche du $49 \$$ que l'on dérive des échelles de sévérité pénales des tribunaux criminels eux-mêmes. Ce tarif informel apparait comme plus "clément" que le tarif législatif officiel (fixé en 1982, il est vrai) qui décrète qu'une amende de $25 \$$ équivaut à une joumée de prison ou à trois heures de travaux compensatoires, lorsque l'amende imposée initialement est de $5000 \$$ ou moins (Loi sur les poursuites sommaires, Lois refondues du Québec, 1983, Annexe A). Les tribunaux criminels consi- 
dèrent, au contraire, qu'un jour de prison équivaut à une amende de $49 \$$ (et pas seulement $25 \$$ ) et que trois heures de travail communautaire valent $52 \$$ d'amende (et pas seulement 30 ).

Le tableau 4 permet de spécifier assez précisément les limites à l'intérieur desquelles on peut envisager de remplacer les peines d'emprisonnement par d'autres sortes de peine. Nous postulons ici que ces peines substitutives doivent posséder le même degré de sévérité que les peines carcérales qu'elles pourraient remplacer. Si ces peines substitutives étaient en réalité perçues comme étant moins sévères que les peines carcérales qu'elles "remplacent", il ne s'agirait plus d'une stratégie d'alternative à l'emprisonnement mais d'une politique d'adoucissement systématique du régime pénal. On doit également prendre en considération les contraintes législatives auxquelles sont assujettis les tribunaux criminels. En supposant un régime législatif dans lequel les maxima sentencielles sont de trois ans pour les peines de probation et de 180 heures pour les mesures de travaux communautaires (régime en vigueur, sauf exception, pour les tribunaux criminels enquêtés dans cette étude), nos données indiquent que de telles peines ne peuvent servir de solution légitime de rechange que pour les sentences carcérales de deux mois et moins (travaux communautaires) ou de 1,3 mois et moins (probation). Le champ d'application de ces peines substitutives est donc passablement limité : elles ne devraient être envisagées que pour des infractions criminelles relativement mineures et pour des accusés sans antécédents judiciaires.

\section{DU RECOURS «EXCESSIF» À L’EMPRISONNEMENT}

Bien que limitée, la marge de manœuvre des peines «substitutives" pourrait l'être davantage si les tribunaux criminels faisaient preuve, comme on le soutient parfois, d'un usage "excessif» de l'emprisonnement. La démonstration de cette thèse ne va pas de soi. Qu'une société comme le Canada par exemple ait un taux d'incarcération qui le situe au sixième rang des 18 pays dont il est fait état dans la dernière édition du Recueil de données concernant les Services correctionnels canadiens ne permet pas de conclure, comme le fait la Commission Archambault $(1987,85,320)$, à un diagnostic de recours excessif à l'emprisonnement: 1) le taux d'emprisonnement per capita constitue une mesure incomplète et inadéquate du taux de punition effectivement infligée parce qu'on ne tient compte ni des régimes de mise en liberté en vigueur ni de la durée des sentences infligées (Downes, 1982; Tremblay, 1986). 2) Toutes choses ne sont pas égales par ailleurs et l'hypothèse du recours "excessif» à l'emprisonnement exige nécessairement que l'on contrôle soigneuse- 
ment et le volume et la gravité des taux de criminalité propres aux sociétés que l'on compare (Steenhuis et coll., 1983). La Suède emprisonne deux fois plus souvent que les Pays-Bas, mais le taux de criminalité y est également deux fois plus élevé. 3) Il est généralement reconnu en sociologie du droit criminel, qu'une société qui choisit de faire un usage relativement parcimonieux de l'emprisonnement "compense" par un contrôle social informel beaucoup plus intense (Black, 1976) ou par un usage plus élevé d'autres formes d'internement (thérapeutique par exemple, Waller, 1982), et vice versa. Il existe donc un prix à payer non seulement lorsque le recours à l'emprisonnement est "trop» élevé mais aussi lorsqu'il ne l'est pas «assez».

Nous proposons ici, à titre heuristique, une deuxième manière de mettre à l'épreuve la thèse du recours excessif à l'emprisonnement en nous basant non pas sur le comportement pénal de diverses sociétés mais sur le comportement sentenciel individuel des acteurs judiciaires euxmêmes. Ceux qui participèrent à notre enquête furent confrontés à cette fin à plusieurs causes criminelles passablement détaillées. Deux d'entre elles, résumées ci-dessous, seront analysées ici:

Cause criminelle $n^{\circ} 1$ : Maurice T., 19 ans, accusé de cambriolage de domicile, commis le jour dans un appartement inoccupé. Vol d'une télévision d'une valeur de $200 \$$. S'enfuit à l'arrivée impromptue du locataire, un dame âgée de 65 ans. Arrêté peu après, aveu immédiat et restitution de l'objet volé. Pas d'antécédents judiciaires, le sujet est étudiant, habite chez ses parents. Père de Maurice T. travaille comme gérant de banque.

Cause criminelle $n^{\circ}$ 2: Brigitte W., caissière de banque, falsifie sa comptabilité et prend dans sa caisse, en une vingtaine d'occasions, des sommes d'argent totalisant $2800 \$$. Agée de 20 ans, toxicomane (héroïne). Dettes de drogues au moment de son arrestation: $4000 \$$. Fréquente un ex-détenu depuis 2 ans. Lorsque arrêtée, elle avoue sur le champ et promet de cesser de prendre de l'héroïne. Pas d'antécédents judiciaires. Vit seule, père chauffeur de taxi et mère travaille dans une manufacture de vêtements.

Le tableau 5 présente non seulement les choix sentenciels des répondants mais une estimation du pourcentage d'acteurs judiciaires "coupables" d'une préférence "excessive» pour l'emprisonnement. Cette estimation requiert que l'on prenne en considération à la fois le taux de change entre peines carcérales et non carcérales et les plafonds que les législateurs imposent en matière de probation et de travaux communautaires. La sévérité perçue d'une peine maximale de travaux communautaires ( 180 heures, en l'occurrence, pour les tribunaux criminels enquêtés 
dans cette étude) équivaut à une peine de 2 mois de prison et une peine maximale de 3 ans de probation "équivaut" à 1,4 mois d'emprisonnement. On peut alors déterminer le pourcentage d'acteurs judiciaires qui, ayant choisi une sentence carcérale de 2 mois et moins, auraient pu, à sévérité égale, remplacer cette sentence par une peine alternative de probation ou de travaux communautaires.

\section{TABLEAU 5}

Choix sentenciels des tribunaux criminels montréalais: estimation de la propension à "préférer. l'emprisonnement à d'autres peines «alternatives"?

\begin{tabular}{|c|c|c|c|c|}
\hline \multirow{2}{*}{$\begin{array}{l}\text { Choix } \\
\text { sentenciels }\end{array}$} & \multicolumn{2}{|c|}{$\begin{array}{l}\text { Cause criminelle no } 1 \\
\text { (cambriolage à domi- } \\
\text { cile, 200\$, pas de } \\
\text { violence, ire con- } \\
\text { damnation, } 19 \text { ans } \\
\text { "Maurice T.") }\end{array}$} & \multicolumn{2}{|c|}{$\begin{array}{l}\text { Cause criminelle } \mathbf{n}^{0} 2 \\
\text { (fraude par caissière } \\
\text { de banque, } 2800 \$, 20 \\
\text { ans, toxicomane, 1re } \\
\text { condamnation } \\
\text { "Brigitte } W . » \text { ) }\end{array}$} \\
\hline & $\mathbf{N}$ & $\%$ & $\mathbf{N}$ & $\%$ \\
\hline Sentences non carcérales & 210 & 89,8 & 55 & 71,4 \\
\hline $\begin{array}{l}\text { Sentences carcérales qui } \\
\text { n'auraient pas pu être } \\
\text { remplacées par des sen- } \\
\text { tences non carcérales }{ }^{2}\end{array}$ & 12 & 5,1 & 18 & 23,4 \\
\hline $\begin{array}{l}\text { Sentences carcérales } \\
\text { qui auraient pu être } \\
\text { remplacées par des sen- } \\
\text { tences non carcérales }\end{array}$ & 12 & 5,1 & 4 & 5,2 \\
\hline Total & 234 & $100 \%$ & 77 & $100 \%$ \\
\hline
\end{tabular}

1 Choix sentenciels de l'ensemble des acteurs judiciaires interrogés, chaque catégorie d'acteur judiciaire (juges, avocats de la défense, procureurs de la Couronne, agents de probation) recevant le même poids.

2 Sentences carcérales de plus de deux mois.

3 Sentences carcérales de deux mois et moins.

Nos résultats indiquent d'abord que la décision de recourir ou non à l'emprisonnement n'est pas particulièrement problématique et qu'elle fait l'objet d'un consensus très général. Par ailleurs, seulement $5 \%$ des répondants ont choisi une sentence d'emprisonnement alors même qu'ils auraient pu choisir - à sévérité égale - une sentence non carcérale. La généralité de la thèse voulant que les tribunaux criminels canadiens 
manifestent une propension "excessive» à recourir à l'emprisonnement doit être, par conséquent, beaucoup plus sérieusement à l'épreuve qu'elle ne l'a été jusqu'à présent. Quant à la minorité de répondants qui ont choisi d'imposer une sentence carcérale de plus de 2 mois d'incarcération, ils n'auraient pas pu recourir aux «alternatives" pénales disponibles, étant donné le taux de change pénal existant et les contraintes législatives en matière de sentences non carcérales.

\section{CONCLUSION}

Nous rappellerons brièvement les principales étapes de l'argumentation développée dans cette étude:

1) Toute stratégie informée d'alternative à l'emprisonnement exige que l'on connaisse au départ la "valeur» des peines ou leur "prix" en termes de sévérité perçue. Nous avons calculé les échelles de sévérité perçues des tribunaux criminels montréalais en matière d'emprisonnement, d'amende, de travaux communautaires et de probation.

2) La manière dont les tribunaux criminels perçoivent la sévérité des peines qu'ils infligent n'expriment pas les préférences idiosyncrasiques des acteurs judiciaires. Il existe, au contraire, un consensus absolu en matière de sévérité perçue des peines entre le public (la société civile) et les tribunaux. Toute réforme sentencielle qui choisit, par ignorance, de sous-estimer ou de surestimer la valeur réelle des peines (en termes de sévérité) se prête aux effets pervers.

3) Une stratégie d'alternative à l'emprisonnement devrait s'assurer que les peines substitutives possèdent le même degré de sévérité que les peines carcérales qu'elles remplacent. Si elles étaient en réalité perçues comme étant moins sévères, alors il ne s'agirait plus «d'alternatives» ou de solutions de rechange. Nous avons donc calculé les équivalences pénales qui peuvent être objectivement établies entre les peines de prison, d'amende, de probation et de travaux communautaires.

4) Le champ d'application des solutions de rechange à l'emprisonnement doit se limiter aux sentences d'emprisonnement de moins de 1,3 mois (ferme) pour la probation et de 2 mois (ferme) pour les travaux communautaires, compte tenu des maxima sentencielles législatives en vigueur ( 3 ans de probation, 180 heures de travaux communautaires dans la province de Québec).

5) Une stratégie de solutions de rechange à l'emprisonnement qui postulerait, au départ, que les tribunaux criminels utilisent les peines 
carcérales plus souvent qu'ils ne devraient théoriquement le faire, doit être accueillie avec scepticisme. Nos résultats suggèrent plutôt que les acteurs judiciaires n'utilisent l'emprisonnement qu'en dernier recours: c'est-à-dire lorsque la sévérité de la sentence qu'ils désirent infliger est trop élevée pour qu'elle puisse être monnayée légitimement en sentences non carcérales (compte tenu, bien entendu, des maximums sentenciels prévus par les législateurs).

\section{RÉFERENCES}

ALLEN, R.B. et R.H. BENSON, "Development of a Punishment Scale: The Item Displacement Phenomenon", Criminal Justice Review, 10, 1985, pp. 39-44.

AUSTIN, J. et B. KRISBERG, "The Unmet Promise of Alternatives to Incarceration", Crime and Delinquency, Juillet 1982, pp. 374-409.

BLACK, D., The Behavior of Law, New York, Academic Press, 1976.

BUCHNER, D., "Scale of Sentence Severity", Journal of Criminal Law and Criminology, $70(2), 1979$, pp. $182-187$.

COUSINEAU, M., D. LABERGE, B. THÉORET, Prisons et prisonniers : une analyse de la détention provinciale québécoise durant la dernière décennie, Montréal, Université du Québec à Montréal, 1986.

CUSSON, M., Pourquoi punir?, Paris, Dalloz, 1987.

DOWNES, D., "The Origins and Consequences of Dutch Penal Policy Since 1945", British Journal of Criminology, 22, 4, 1982, pp. 325-362.

ERICKSON, M.L. et J.P. GIBBS, "On the Perceived Severity of Legal Penalties" , Journal of Criminal Law and Criminology, 70, 1979, pp. 102-116.

HAMBLIN, R.L., "Social Attitudes: Magnitude Measurement and Theory", dans H.M. Blalock (édit.), Measurement in the Social Sciences. Chicago, Aldine, 1974.

HUDSON, J. et S. CHESNEY, "Research in Restitution: A Review and Assessment", dans B. Galaway, J. Hudson (édit.), Offender Restitution in Theory and Action, Lexington, Lexington Books, 1978.

JACOBSEN, C., T. VAN DER VOORDT, «Interpreting Modal Frequencies to Measure Social Norms", Sociological Methods and Research, 8, 4, 1980, pp. 470-486.

LODGE, M., Magnitude Scaling : Quantitative Measurement of Opinions, Beverly Hills, Sage, 1981 .

McCLELLAND, K.A. et G.P. ALPERT, «Factor Analysis Applied to Magnitude Estimates of Punishment Seriousness: Patterns of Individual Differences», Journal of Quantitative Criminology, 1(3), 1985, pp. 307-318.

MIETHE, T.D., «Types of Consensus in Public Evaluations of Crime : An Illustration of Strategies for Measuring Consensus", Journal of Criminal Law and Criminology, 75, 2,1984 , pp. $459-473$.

NAMBOODIRI, K. et L. CARTER, H. BLALOCK, Applied Multivariate Analysis and Experimental Design, New York, McGraw Hill, 1975. 
NEWMAN, G., Just and Painful : A Case for the Corporal Punishment of Criminals. New York, Macmillan, 1983.

PEASE, K., "Community Service Orders», dans M. Tonry, N. Morris (édit.), Crime and Justice: An Annual Review of Research, vol. 6, Chicago, University Press, 1985.

ROSSI, P.H. et R.A. BERK, „Varieties of Normative Consensus», American Sociological Review, 50, Juin 1985, pp. 333-347.

ROSSI, P.H. et J.E. SIMPSON, J. MILLER, «Beyond Crime Seriousness: Fitting the Punishment to the Crime", Journal of Quantitative Criminology, 1, 1985, pp. 59-90.

SEBBA, L., \&Some Explorations in the Scaling of Penalties", Journal of Research in Crime and Delinquency, 15, 1978, pp. 247-265.

SEBBA, L. et G. NATHAN, «Further Explorations in the Scaling of Penalties», British Journal of Criminology, 23(3), 1984, pp. 221-249.

SHINN, A., "Relations between Scales", dans H.M. Blalock (édit.), Measurement in the Social Sciences, Chicago, Aldine, 1974.

SIMON, $\mathrm{H}$, et $\mathrm{S}$. VALLIÈRES, "L'évaluation d'une nouvelle mesure pénale: les travaux communautaires au Québec" Cahiers de l'École de criminologie, Montréal, Université de Montréal, 1982.

STEENHUIS, D.W., L.C.M. TIGGES, J.J.A. ESSERS, "The Penal Climate in the Netherlands: Sunny or Cloudy?», British Journal of Criminology, 23, 1, 1983, pp. 1-16.

STEVENS, S.S., Psychophysics : Introduction to its Perceptual, Neural and Social Prospects, New York, Wiley, 1975.

TREMBLAY, P., "The Stability of Punishment: A Follow-Up of Blumstein's Hypothesis", Journal of Quantitative Criminology, 2, 2, 1986, pp. 257-280.

TREMBLAY, P., "On Penal Metrics: Pursuing Erickson and Gibbs's Hypothesis», Journal of Criminal and Criminology, (à paraitre, 1987).

WALLER, I., Canadian Crime and Justice in Comparative Perspective: Selected Indicators for Selected Countries, 1900-1980. Unpublished Research, Criminology Department, University of Ottawa, Ottawa.

WOLFGANG, M.E., R.M. FIGLIO, P.E. TRACY et S.I. SINGER, The National Survey of Crime Severity. Washington, U.S. Department of Justice, 1985. 